\title{
L'application des connaissances sur l'ostéoporose chez les jeunes adultes : nouvelles orientations pour les programmes de prévention
}

\begin{abstract}
Alyson Holland, Ph. D.
Cet article a fait l'objet d'une évaluation par les pairs.

\section{Résumé}

Introduction : La prévention de l'ostéoporose dépend grandement des programmes d'éducation, qui sont efficaces surtout lorsqu'ils sont adaptés à leurs destinataires. La plupart des programmes d'éducation préventive étant conçus pour les adultes relativement âgés, leur application est plus difficile auprès d'un public adulte plus jeune. Concevoir des programmes à l'intention des jeunes adultes nécessite de tenir compte de leurs pratiques de recherche d'information, de manière à favoriser une application efficace des connaissances sur l'ostéoporose.
\end{abstract}

Méthodologie : Des entrevues individuelles ont été réalisées auprès de 60 hommes et femmes (population multiethnique composée de jeunes adultes canadiens) pour connaître leurs sources d'information et les types de renseignements recherchés en matière de nutrition ou de santé des os.

Résultats : Les résultats de notre étude portent sur les sources utilisées par les participants, leurs champs d'intérêt et les façons de mobiliser les jeunes adultes. Les programmes de prévention devraient exploiter à la fois les sources d'information traditionnelles, telles que les pairs, les membres de la famille et les professionnels de la santé, et les nouvelles technologies, comme les médias sociaux. Le choix des sources s'est révélé motivé par leur autorité perçue et la confiance qui leur était accordée. L'information devrait rejoindre les champs d'intérêt des jeunes adultes et les thèmes sur lesquels ils s'informent déjà, comme le conditionnement physique et l'alimentation, plutôt qu'être intégrée exclusivement à une documentation de sensibilisation sur l'ostéoporose. On peut susciter l'intérêt des jeunes adultes à l'aide de messages courts auxquels ils peuvent s'identifier et qui les encouragent à effectuer de petits changements dans leurs habitudes. Les intérêts en matière d'information variaient légèrement en fonction du sexe des participants. Les différences attribuables à l'âge des participants n'ont pas été examinées.

Conclusion : La manière la plus susceptible d'encourager les jeunes adultes à adopter des comportements de prévention consiste à créer de courts messages axés sur l'action, visant à les amener à réaliser de petits changements de comportement et contenant des renseignements en lien avec leurs recherches actives.

Mots-clés : ostéoporose, éducation préventive, jeunes adultes, méthodes qualitatives

\section{Introduction}

L'ostéoporose est la maladie métabolique des os la plus courante : elle touche près de 2 millions de Canadiens et près de 200 millions de personnes dans le monde ${ }^{1,2}$. Actuellement, une femme sur trois et un homme sur cinq en souffrent au Canada, et la maladie apparaît généralement au cours de la cinquantaine ${ }^{3}$. L'ostéoporose a de graves conséquences, en particulier des fractures (de la hanche, de la colonne vertébrale et des poignets), qui sont associées à des taux de morbidité et de mortalité accrus $^{2}$. L'ostéoporose entraîne des douleurs chroniques, une hausse du taux
Points saillants

- Une application efficace des connaissances implique d'utiliser les réseaux sociaux traditionnels et émergents.

- Les jeunes adultes ne se préoccupent pas des maladies risquant de les toucher plus tard. L'information diffusée doit donc relever de leurs champs d'intérêt du moment en matière d'apparence et de conditionnement physique.

- Pour être efficaces, les messages de promotion de la santé devraient utiliser des formulations positives afin de promouvoir de légers changements à intégrer au quotidien en matière d'habitudes alimentaires et de choix des aliments.

d'hospitalisation ainsi qu'une perte d'autonomie et d'indépendance ${ }^{4}$. Elle est donc considérée comme un problème de santé publique important ${ }^{5}$. Les coûts élevés relatifs à l'ostéoporose, estimés à 2,3 milliards de dollars par année au Canada, combinés à une incidence accrue de la maladie, font qu'on accorde maintenant davantage d'importance aux mesures de prévention, dans le but de réduire la perte osseuse et de prévenir ainsi les fractures ${ }^{2}$. La prévention de l'ostéoporose repose en grande partie sur l'éducation, dans le but d'éviter une future hausse de la prévalence $^{6,7}$. Cette éducation préventive comprend surtout de l'information sur l'activité physique et la nutrition, cette dernière étant présentée comme une habitude de vie relativement simple à changer ${ }^{8}$.

Rattachement de l'auteure :

Département d'anthropologie, Université McMaster, Hamilton (Ontario), Canada

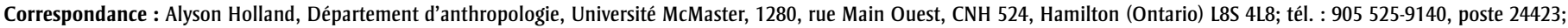
courriel : jaagumae@mcmaster.ca 
L'éducation préventive de l'ostéoporose repose sur une application efficace des connaissances, qui se définit comme la synthèse, la dissémination, l'échange et l'application conforme à l'éthique des connaissances dans le but d'améliorer la santé des personnes et l'efficacité du système de santé 9 . On peut conceptualiser le processus d'application des connaissances à l'aide du cycle de mise en pratique, qui présente l'application efficace des connaissances comme un processus dont l'aboutissement est la mise en pratique continue de l'information obtenue par les utilisateurs de connaissances ${ }^{10,11}$. Ce cycle consiste à repérer les lacunes dans les connaissances, à comprendre les pratiques de recherche de renseignements du public cible et les obstacles qui limitent l'accès à ces renseignements, à concevoir de l'information qui comble les lacunes identifiées tout en demeurant compréhensible, recherchée et assimilable pour le public cible ${ }^{11}$.

$\mathrm{Au}$ Canada, l'éducation préventive est coordonnée au niveau national par Ostéoporose Canada, une organisation non gouvernementale qui vise à améliorer la prévention et la gestion de l'ostéoporose. Bien qu'Ostéoporose Canada constitue la source principale des messages portant sur l'ostéoporose, il n'existe aucune stratégie nationale de mise en place de pratiques exemplaires en la matière. Chaque province a donc conçu son propre plan d'action de lutte contre l'ostéoporose*. En 2005, l'Ontario a ainsi mis en place sa stratégie de lutte contre l'ostéoporose (Ontario Osteorporosis Strategy, ou OOS), conçue pour prévenir et gérer l'ostéoporose au sein de la province, qui figure parmi les plus peuplées du Canada. Ce programme consiste en une approche multifactorielle axée sur la prévention des fractures, sur l'éducation des patients et des professionnels de la santé, sur la réduction des risques de chute et sur les pratiques d'autogestion des patients afin de réduire les répercussions de l'ostéoporose. La stratégie de lutte contre l'ostéoporose de l'Ontario est principalement conçue pour les adultes relativement âgés dans la mesure où ce sont eux qui présentent les symptômes associés à la maladie ${ }^{16}$. La mise en œuvre du programme est assurée par la création de cliniques régionales d'ostéoporose et de fractures, qui offrent des traitements adaptés aux personnes ayant une faible densité osseuse ou ayant subi des fractures de fragilisation, et par la prestation à grande échelle de programmes de prévention des fractures par Ostéoporose Canada $^{17}$. Les programmes d'éducation préventive de la stratégie OOS visent à agir sur certaines facettes du style de vie des adultes plus âgés, comme la prévention des chutes, les changements d'habitudes alimentaires en raison d'affections concomitantes, la diminution de l'apport alimentaire liée à l'âge, les interventions pharmacologiques et l'activité physique à faible impact $^{18}$. Les jeunes adultes, définis ici comme étant âgés de 17 à 30 ans, période au cours de laquelle les habitudes alimentaires d'adulte et la masse osseuse se développent, ne sont pas touchés par cette information préventive, car leurs préoccupations et leurs champs d'intérêt diffèrent de ceux des adultes plus âgés ${ }^{9,19,20}$. Les contraintes financières des organisations sans but lucratif, qui assurent la majeure partie de la tâche de diffusion de l'information, ont une influence sur la portée des programmes de sensibilisation sur l'ostéoporose : comme il est nécessaire de viser surtout les personnes les plus à risque, les jeunes adultes ne sont souvent pas pris en compte. Or, bien que ces derniers n'aient pas de risque élevé de développer de l'ostéoporose, leurs habitudes alimentaires peuvent avoir une influence sur la survenue de la maladie, ce qui veut dire que la prévention précoce auprès de ce groupe est nécessaire pour réduire la prévalence future de l'ostéoporose $^{21}$. La raison pour laquelle les institutions gouvernementales n'accordent que très peu d'importance à l'éducation sur l'ostéoporose auprès des jeunes adultes n'est pas claire. Alors que la stratégie de lutte contre l'ostéoporose de l'Ontario souligne l'importance de l'éducation à tous les âges pour préserver la santé des os, seules les classes d'élèves de quatrième année ont bénéficié de ses programmes ${ }^{12}$. Même si la stratégie de lutte contre l'ostéoporose de l'Ontario a été mise en place il y a plus de dix ans, elle n'a fait l'objet que d'une étude évaluative publiée ${ }^{17}$, ce qui veut dire que l'état actuel de l'application des connaissances sur l'ostéoporose en Ontario est mal connu. L'information à ce sujet a surtout été axée sur l'évaluation des connaissances des adultes plus âgés et sur les types de fractures qu'ils subissent, sans qu'on se soit penché sur les façons dont les populations plus jeunes accèdent à l'information sur l'ostéoporose et l'assimilent ${ }^{22,23}$.

L'Enquête sur la santé dans les collectivités canadiennes de 2004 indique que la consommation chez les jeunes adultes de calcium et de vitamine $\mathrm{D}$, nutriments essentiels à la santé des os, demeure faible, ce qui augmente leurs risques de développer de l'ostéoporose et d'autres affections des $\mathrm{os}^{24}$. Si l'on mise sur l'éducation préventive sur l'ostéoporose pour augmenter la consommation de calcium et de vitamine D chez les jeunes adultes, il importe de concevoir des programmes éducatifs adaptés à leur style de vie et à leurs pratiques de recherche de renseignements ${ }^{20}$. Bien que la littérature fasse état d'une base de connaissances sur l'ostéoporose chez les jeunes adultes $^{25-27}$, leur processus d'acquisition et d'application de ces connaissances reste vague. Notre étude se penche sur les sources et les types de renseignements que les jeunes adultes consultent lorsqu'ils se renseignent sur la nutrition et la santé des os, dans le but de permettre l'élaboration de stratégies visant à assurer une application plus efficace des connaissances dans les programmes d'éducation sur l'ostéoporose.

\section{Méthodologie}

Dans le cadre de cette étude, des entrevues individuelles semi-dirigées en profondeur ont été réalisées afin d'explorer les comportements de recherche d'information sur l'ostéoporose chez les jeunes adultes canadiens. Les entrevues ont été menées entre septembre 2013 et juin 2014 auprès de 60 personnes ( 30 hommes et 30 femmes) d'origines ethniques variées. Les participants étaient de jeunes adultes âgés de 17 à 30 ans vivant dans la grande région de Hamilton (Ontario). Le groupe d'âge de ces participants constitue une interprétation large de la notion de «jeune adulte» utilisée dans la littérature ${ }^{28,29}$ et a été choisi parce qu'il permet d'inclure les étudiants et les jeunes professionnels, qui développent leurs habitudes alimentaires en tant qu'adultes indépendants et chez qui les os continuent de croître ${ }^{19,21}$. Les renseignements concernant l'affiliation ethnoculturelle ont été recueillis afin de s'assurer que les participants soient représentatifs de la diversité de population de Hamilton. On

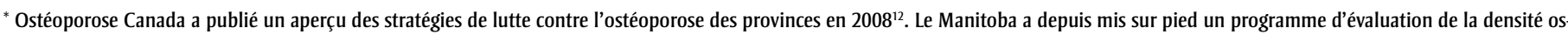

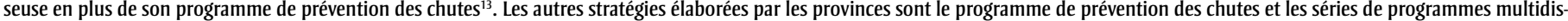

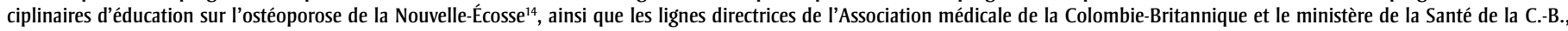
publiées en 2012 concernant le diagnostic, la prévention et le traitement de l'ostéoporose ${ }^{15}$.
} 
a demandé aux participants d'indiquer leur affiliation ethnoculturelle, ce qui a créé un large éventail de réponses impossibles à classer en catégories de base.

Les participants affichaient des niveaux de scolarité et des statuts socioéconomiques variés. Leur recrutement a été effectué à l'Université McMaster (étudiants inscrits à un programme de quatre ans), au Mohawk College (étudiants inscrits à un programme de deux ans) et localement. Les participants locaux comprenaient des diplômés postsecondaires et des étudiants sans formation postsecondaire (dont des étudiants et des diplômés du secondaire qui travaillaient ou qui étaient sans emploi). Les participants potentiels ont été recrutés à l'aide d'affiches sur le campus et à Hamilton, ainsi qu'au moyen des médias sociaux (Facebook).

Les participants devaient faire partie du groupe d'âge sélectionné, vivre à Hamilton et être en mesure de donner leur consentement. L'approbation éthique a été obtenue auprès des comités d'éthique de la recherche de l'Université McMaster et du Mohawk College. Le consentement écrit a été obtenu avant la participation à l'étude.

Les participants devaient remplir un questionnaire sociodémographique et participer à une entrevue individuelle. Le questionnaire visait à recueillir des renseignements concernant l'âge, le sexe, le revenu, l'éducation, l'emploi et l'origine ethnoculturelle. Les entrevues ont été menées sur le campus ou dans des lieux publics accessibles (p. ex. bibliothèque, YMCA) et ont duré entre 60 et 90 minutes chacune. Toutes ont été réalisées, enregistrées et transcrites textuellement par l'auteur. On a posé aux participants des questions sur principalement trois sujets : leurs attitudes à l'égard des activités liées à la nutrition et à la santé des os et leur participation à ces dernières (p. ex. "Quelle importance accordez-vous à la nutrition et à la santé des os dans votre quotidien? »), leurs pratiques de recherche d'information sur la santé et sur la nutrition (p. ex. "Que consultez-vous pour trouver des renseignements sur la santé des os et sur la nutrition? ») et leurs champs d'intérêt liés à la santé et à la nutrition (p. ex. " Qu'est-ce qui vous intéresse au sujet de la santé des os et de la nutrition? »). (Le guide d'entrevue complet peut être obtenu sur demande auprès de l'auteur.) Les participants devaient également suggérer des façons d'améliorer les messages de santé à l'intention des jeunes adultes. Les entrevues étaient semi-dirigées, et tous les participants ont répondu aux mêmes questions tirées du guide d'entrevue. Des questions supplémentaires variant selon les réponses reçues ont été posées pour obtenir davantage de renseignements. Les participants pouvaient orienter la conversation, ce qui explique les réponses variées aux questions d'entrevue. Cette étude relève d'un projet de doctorat plus large sur la motivation des jeunes adultes à l'égard de l'éducation sur la prévention de l'ostéoporose axée sur la nutrition, l'objectif du projet étant de déterminer comment les connaissances sur la nutrition et sur les risques de maladie sont acquises. Une analyse détaillée de la méthodologie et des questions de recherche est disponible ailleurs ${ }^{30}$.

La transcription des entrevues a été réalisée avec la version 10 du logiciel NVivo (QSR International Pty, Melbourne, Australie) afin de classer les codes et de déceler les liens entre thèmes. Une analyse de contenu thématique a été réalisée pour identifier les thèmes manifestes (explicites) et latents qui ressortaient des données obtenues et qui rendaient compte des significations, des expériences et des réalités exprimées par les participants ${ }^{31}$. Les thèmes ont été définis conformément au processus décrit par Bernard ${ }^{32}$ pour l'analyse qualitative et théorique du contenu en anthropologie. Les codes ont été élaborés sur la base d'une approche théorique selon laquelle la question de recherche sert à orienter l'analyse des données. Comme ce projet faisait partie d'une étude plus vaste, les données ont été analysées selon certaines questions de recherche spécifiques. Le guide d'entrevue a été conçu pour examiner des thèmes précis plutôt que pour étudier un phénomène de manière générale. En l'occurrence, la question de recherche portait sur la compréhension du processus par lequel les jeunes adultes cherchent à acquérir des connaissances axées sur la nutrition et sur la santé.

Le codage a été effectué en deux étapes. Dans un premier temps, on a utilisé des codes préétablis relatifs à la question de recherche (motivation, connaissances, sources), ce qui a créé de grandes catégories. En second lieu, on a effectué le codage de ces grandes catégories sans code préétabli. Les thèmes ont plutôt été déterminés et codés lorsqu'ils émergeaient du texte ${ }^{32}$, ce qui a permis à des sous-thèmes plus nuancés d'apparaître. On a identifié les thèmes mentionnés explicitement par les participants ainsi que les thèmes latents apparus à la suite d'une lecture attentive et de l'interprétation des données. Chaque transcription a été lue à deux reprises par l'auteur afin de s'assurer que tous les codes possibles étaient attribués. Une fois générés, les codes ont été hiérarchisés à l'aide de NVivo 10 de façon à refléter les thèmes dominants, puis ont été fusionnés afin d'éviter une redondance des concepts. Le codage a été réalisé en six mois, et l'ensemble des données a été soumis à une réflexion de fond dans le but d'éviter les biais. L'auteur a effectué le codage et l'analyse seule.

Les participants ont été répartis par sexe afin d'explorer les différences potentielles entre les pratiques de recherche d'information liée à la santé en fonction du sexe. Aucune analyse en fonction de l'âge n'a été possible en raison du petit nombre de participants de chaque groupe d'âge. Les subdivisions au sein du groupe d'âge des jeunes adultes n'ont présenté aucune différence significative. Aucune analyse en fonction de l'ethnicité n'a été possible en raison des multiples identités ethnoculturelles déclarées par les participants. On a cependant pu examiner les grandes tendances quant au choix des sources et aux champs d'intérêt en matière de santé et de nutrition.

\section{Résultats}

\section{Résultats généraux}

Trois sujets principaux de discussion émergent des entrevues : les sources d'information, les champs d'intérêt liés à la santé et à la nutrition et les suggestions pour motiver les jeunes adultes à chercher de l'information sur la santé et sur la nutrition. Les sources d'information constituent l'élément primordial pour l'application des connaissances, puisque c'est par elles que les participants ont reçu l'information, que ce soit de manière active ou passive. Les participants recherchaient rarement de l'information sur la santé ou sur la nutrition consciemment, mais ils savaient tout de même repérer les réseaux fiables par lesquels ils recueillaient au besoin de l'information. Le degré d'assimilation de l'information tirée de ces sources était directement lié à leurs champs d'intérêt en matière de santé et de nutrition : ceux-ci déterminaient le type d'information recherché et les sources précises consultées. 
Toutes les croyances des participants en matière de nutrition correspondaient aux discours réductionnistes des sources faisant autorité dans le domaine, mais euxmêmes étaient plutôt personnellement motivés par leur environnement social et leur corps. Les suggestions qu'ils ont proposées pour améliorer l'application des connaissances étaient de ce fait la création de programmes d'éducation qui tiennent compte de ce qui intéresse vraiment les jeunes adultes en matière de santé et de nutrition, qui captent leur attention et qui correspondent à leurs motivations réelles.

\section{Sources d'information}

Les participants ont recensé un large éventail de sources consultées lors de leur recherche ou découverte d'information sur la nutrition : les médecins et les autres professionnels de la santé, les parents, la famille, les amis, le gouvernement, les étiquettes des aliments, les revues, les annonces publicitaires, les livres, l'école, les journaux, l'Église et Internet, en particulier les médias sociaux. Par exemple, un participant a déclaré : "Vous savez, je parlerais probablement à mes parents en premier pour avoir leur avis et je m'en servirais comme information de base. J'irais voir mon médecin et je lui dirais "voici ce que je pense” et je partirais de ça. " (homme, 19 ans, étudiant au College, participant [P] 45). Les sources d'information les plus couramment citées ont été Internet, le gouvernement, les médecins, les amis et les parents (tableau 1). La plupart des participants ont relevé deux ou trois sources consultées régulièrement. Leur recherche d'information se faisait en grande partie en ligne, mais ils ont indiqué que les réseaux traditionnels (parents et pairs) demeuraient également des sources importantes. Aucune différence importante n'a été constatée entre les sources utilisées par les participants des deux extrémités du groupe d'âge, ce qui indique que ces sources sont consultées par tous les jeunes adultes.

En général, l'évaluation des sources effectuée par les participants semblait hautement subjective et liée à des idées préconçues : «Je fais quelques recherches si ça semble peu vraisemblable. Mais si c'est devant moi, que je suis quand même d'accord avec ce que je lis, alors oui. » (femme, 28 ans, diplômée, P 77). La plupart des participants préféraient les sources fournissant de l'information allant dans
TABLEAU 1

Sources d'information privilégiées par les jeunes adultes (17 à 30 ans) en matière de santé des os et d'alimentation, Hamilton, Ontario, 2013-2014

\begin{tabular}{lc} 
Sources & $\begin{array}{c}\text { Nombre de répondants } \\
(\mathbf{N}=\mathbf{6 0})\end{array}$ \\
\hline Internet & 57 \\
Gouvernement & 11 \\
Médecins & 25 \\
Amis & 18 \\
Parents & 15 \\
Établissement scolaire & 7 \\
Pharmaciens & 6 \\
Magazines & 6 \\
Télévision & 3 \\
Livres & 2 \\
Journaux & 2 \\
Lieu de culte & 1 \\
\hline
\end{tabular}

le sens de leurs opinions et de leurs comportements.

Presque tous les participants ont évalué les sources selon leur fiabilité (p. ex. " [Les médias sociaux] ne sont pas fiables, ce n'est que l'opinion de quelqu'un. » [homme, 29 ans, étudiant à l'université, P 8]), selon l'autorité perçue (p. ex. "Les célébrités en savent davantage sur la nutrition. " [femme, 21 ans, étudiante à l'université, P 5]) et selon le niveau de confiance accordé à la source (p. ex. " J'essaie de consulter le magazine Men's Health parce que je sais qu'il est digne de confiance. Certains conseils que j'ai suivis m’ont vraiment été utiles. " [homme, 18 ans, étudiant au secondaire, P 80]). Les sources considérées par les participants comme documentées et fiables, par exemple les parents, ont été perçues implicitement comme dignes de confiance. De plus, la plupart des participants étaient moins susceptibles d'exiger des preuves ou une vérification supplémentaire des faits. Les parents ont une connaissance approfondie de leurs enfants, et c'est peut-être pourquoi les participants choisissaient souvent de les consulter. " J'irais voir mon père parce qu'il y a tellement de renseignements contradictoires sur Internet et qu'il me connaît assez pour dire "Je sais ce que tu veux accomplir, et ceci t'aidera à atteindre ton objectif”. " (homme, 28 ans, étudiant à l'université, P 28).
Les participants qui ont mentionné les professionnels de la santé comme source les considéraient comme étant bien documentés et fiables, présumant qu'ils possédaient de bonnes connaissances. "Mon médecin me dit quoi faire, alors je fais ce qu'il dit parce que c'est un médecin. " (homme, 18 ans, étudiant à l'université, P 14). Les médecins étaient en particulier plus souvent mentionnés comme source préférentielle d'information par les femmes, même si les hommes les considéraient également comme importants.

Tous les participants qui ont consulté des amis ou des membres de leur famille (excepté leurs parents) ont choisi ceux qui pouvaient leur fournir des preuves scientifiques ou anecdotiques de leurs connaissances dans le domaine. En général, la personne devait démontrer qu'elle adoptait un régime alimentaire nourrissant et des comportements perçus comme sains ou qu'elle avait suivi une formation dans un domaine de la santé afin d'être considérée comme une bonne source. "Je dirais que c'est surtout du bouche-à-oreille provenant de personnes qui sont plus éduquées que moi dans le domaine. Alors je dirais que ma mère, mon entraîneur et mes coéquipiers sont des sources importantes. » (femme, 23 ans, étudiante à l'université, P 15).

Les participants ont fréquemment relevé que les sources devaient fournir des preuves ou relever d'une affiliation professionnelle. Les sources en ligne étaient particulièrement soumises à ces exigences et devaient fournir des preuves ou des références (p. ex. «Je consulte des blogues et des trucs du genre, mais j'essaie de trouver des références pour confirmer ce qui y est dit. » [femme, 30 ans, étudiante à l'université, P 3]), être confirmées par un professionnel de la santé ou être associées à une institution gouvernementale (p. ex. " J'irais visiter des sites qui sont un peu plus valables, comme les sites du gouvernement sur la nutrition. " [femme, 24 ans, étudiante à l'université, P 4]). Cependant, un petit nombre de participants ( $n=6$ ) n'exigeaient pas le même degré de validité de l'information et choisissaient simplement les résultats les plus courants trouvés sur Internet. " Je consulte les dix meilleurs résultats, et si cinq ou six d'entre eux indiquent qu'il faut manger des bleuets, je suis habituellement le conseil et j'en mange. » (homme, 20 ans, étudiant au College, P 69). 
Les médias sociaux en tant que source en ligne étaient traités un peu différemment. Tous les participants, sauf quatre, ont affirmé ne pas avoir cherché activement de renseignements concernant la santé ou la nutrition sur les médias sociaux. Toutefois, lorsqu'ils ont été questionnés davantage, ils ont mentionné qu'ils lisaient souvent l'information relayée par les médias sociaux (p. ex. sur un fil Twitter ou Facebook) lorsqu'elle semblait intéressante. Cette attitude envers les médias sociaux a été résumée par un participant qui a dit à propos de Facebook : " Je vois passer l'information sur le fil d'actualité, mais je ne l'ai pas vraiment cherchée. De temps en temps, lorsque quelque chose attire mon attention, je lis l'article ou la nouvelle en entier. » (homme, 28 ans, étudiant au College, P 73).

Les quatre participants qui ont utilisé les médias sociaux comme source ont suivi des professionnels de la santé et des amis pour obtenir davantage d'information sur la santé ou ont posé des questions sur ces plates-formes pour obtenir des réponses de ceux qu'ils considéraient comme informés. " Je trouve beaucoup de renseignements sur les médias sociaux et parfois des personnes ou des amis peuvent avoir des idées auxquelles je n'avais jamais pensé. » (homme, 19 ans, étudiant au College, $P$ 45). Les médias sociaux ont été perçus comme une piètre source d'information par la plupart des participants (parce qu'ils ne sont pas fiables et qu'ils sont fondés sur des opinions), mais tous sauf deux les utilisaient chaque jour. Les participants étaient donc exposés de façon passive à de l'information sur la santé et sur la nutrition qu'ils n'auraient pas cherchée autrement.

\section{Champs d'intérêt liés à la santé et à la nutrition}

La plupart des participants ont considéré la santé et la nutrition comme des concepts différents, sans cependant que ce soit explicite. Le thème de la recherche sur la santé a émergé principalement lors des discussions sur les symptômes de maladie, alors qu'il a été question de nutrition plutôt dans les discussions en vue d'améliorer la santé. Presque tous les participants ont indiqué avoir fait des recherches précises uniquement pour de l'information médicale, lorsqu'ils vérifiaient des symptômes, et non pour de l'information nutritionnelle. Ils ont généralement utilisé pour ce faire des sites traitant de la vérification des symptômes (p. ex. WebMD). « Je sais que tout le monde dit qu'il faut regarder dans Google et y taper ce qu'on cherche, et que ça dit que tu vas mourir. Je commencerais par là, puis j'essaierais de connaître les opinions des gens, de savoir ce qu'ils pensent et j'irais voir mon médecin par la suite. " (homme, 18 ans, étudiant à l'université, P 14) Les participants estimaient que les sites traitant de la santé encourageaient les discours alarmistes et qu'il n'y avait pas lieu de rechercher des renseignements sur des questions médicales sauf en cas de problème précis. L'intérêt général pour l'obtention de renseignements médicaux n'augmentait pas avec l'âge, mais était fonction de problèmes à résoudre. On a constaté que faire de la recherche active et obtenir des renseignements sur la santé avait fait l'objet d'un intérêt plus grand chez les participants de tous âges touchés antérieurement par des problèmes de santé, ce qui révèle que l'expérience a une influence importante sur la motivation.

Douze participants ont déclaré n'avoir aucun intérêt envers l'alimentation ni la nutrition. Chez les autres, les raisons incitant à effectuer des recherches peuvent être regroupées en trois catégories : pour savoir quoi manger afin d'atteindre un objectif précis, pour acquérir des connaissances sur le contenu des aliments et enfin pour apprendre des faits sur l'alimentation ou suivre des titres accrocheurs. Tous les participants sauf trois ont indiqué chercher de l'information nutritionnelle de façon irrégulière, mais en trouver souvent à la suite de comportements liés à la recherche d'information. "Je ne cherche pas vraiment le genre de choses dont j'ai besoin de prendre en ligne. Je dirais juste le genre d'aliments dont on aurait besoin si on était dans telle ou telle situation. " (femme, 21 ans, étudiante à l'université, P 05). Pour la plupart des participants, lire sur l'alimentation s'est révélé un des moyens de parvenir à perdre du poids ou à améliorer sa condition physique et sa santé en général, à élaborer un régime alimentaire plus sain afin d'éviter les maladies, à réduire les coûts liés à l'alimentation, à trouver des recettes et à atténuer la peur de la maladie. Les objectifs liés à la perte de poids et à la condition physique étaient souvent ce qui motivait les changements dans les habitudes alimentaires. " [Je cherche] à savoir quelles sont les vitamines contenues en grande quantité dans un aliment, combien a-t-il de calories, parce qu'en sachant le genre de vitamines qu'il comprend, ça me sert de guide en quelque sorte pour savoir le type d'exercices que je devrais faire, comment est-ce que ça pourra optimiser ma mobilité physique. » (homme, 22 ans, étudiant au College, P 42). Les participants étaient plus susceptibles de rechercher des renseignements ou de suivre des liens traitant de l'amélioration de la force $(\mathrm{n}=15)$ ou de la perte de poids ( $\mathrm{n}=23$ ). Si les hommes comme les femmes s'intéressaient à la condition physique et à la perte de poids, les hommes ont mentionné plus souvent que la condition physique constituait un objectif, tandis que c'était la perte de poids ou l'apparence pour les femmes. Les recherches en matière de saine alimentation étaient aussi associées à des intentions d'amincissement et de renforcement du corps.

Les participants étaient également intéressés par le contenu des aliments qu'ils consommaient. Pour un petit nombre de participants, la recherche des ingrédients des aliments préparés ou des nutriments des aliments entiers était motivée par un intérêt pour les politiques alimentaires et la science de l'alimentation. Pour une plus grande proportion de participants, il s'agissait de s'informer sur les ingrédients des aliments dans le but d'améliorer sa condition physique, perdre du poids et adopter une alimentation saine de sorte à éviter les maladies. L'origine des produits alimentaires ainsi que les influences exactes des produits chimiques ajoutés ou des nutriments sur l'organisme se sont révélées particulièrement importantes pour les participants.

Je ne sais pas, c'était juste comme, c'est une bonne chose, je suppose. C'est bien de savoir ce que tu donnes à ton organisme et d'en être conscient. Je crois que, de manière générale, c'est une prise de conscience de ce que tu manges, c'est ta santé, tu dois veiller à ton bien-être. Je pense que, pour moi, c'est une prise de conscience de ce qui existe, de ce qui est bon, et peut-être de ce qui est mieux encore; et peut-être de ce qui n'est pas très bon, mais qui est commercialisé comme étant bon. (femme, 23 ans, diplômée, P 55)

Comme cette participante l'a déclaré, les champs d'intérêt en matière d'alimentation sont liés à la santé, au corps et à un désir grandissant de prendre en main sa santé. La plupart des participants ont mentionné qu'il était important d'avoir des connaissances à 
propos de ce qu'ils consommaient de manière à être conscients de l'équilibre entre les "bons " et les " mauvais " aliments ingérés. En effectuant leurs recherches, ces participants avaient l'impression de prendre en charge leur santé activement et de faire des choix éclairés. Au lieu de s'informer uniquement à propos des aliments qu'ils devaient manger, les participants souhaitaient aller plus loin et cherchaient à s'informer sur ce qui pouvait les motiver à les consommer. "[Je veux savoir] dans quelle mesure certains aliments me seraient bénéfiques et pourquoi il est important d'ingérer certaines choses. » (femme, 22 ans, diplômée, P 43). Le désir d'être actifs et de prendre en charge leur santé a conduit les participants à chercher plus de renseignements sur leur alimentation pour découvrir les raisons pour lesquelles consommer certains aliments et les effets de ces derniers sur l'organisme.

La dernière raison pour laquelle les participants cherchaient des renseignements sur l'alimentation relève d'un intérêt passif les conduisant vers des liens dont les titres d'articles étaient intrigants ou vers des faits sur l'alimentation. Ces participants ( $\mathrm{n}=16$ ) cliquaient généralement sur des liens présentant des déclarations inhabituelles, de l'information contradictoire ou des faits intéressants sur l'alimentation. "J'aime beaucoup quand je lis "saviez-vous que" et des trucs, genre, trouvés au hasard. Ça peut être quelque chose que tu ne savais pas, comme "savais-tu que cet aliment contenait tous ces types de nutriments et qu'il est bon pour ceci, cela et ceci. Voilà ce que ça fait à ton organisme”. " (femme, 18 ans, étudiante à l'université, P 2). La plupart ne cherchaient pas d'information sur l'alimentation de façon active, mais ont été motivés par ce qu'ils lisaient. "Les articles de Yahoo disent genre "les cinq aliments dont vous avez besoin tous les jours”, ou quelque chose comme ça. Alors tu les lis, genre, et ça te fait réfléchir, genre : “Est-ce que j'ai vraiment besoin de ça? Est-ce que j'en ai mangé dernièrement?" Puis, si ça te fait penser à autre chose, tu vas commencer à regarder ailleurs. " (homme, 21 ans, étudiant à l'université, P 6). Ces recherches étaient le plus souvent associées aux médias sociaux, faisant suite aux liens affichés par les amis ou connaissances et non à un questionnement actif sur l'alimentation.
Sensibiliser les jeunes adultes à l'information liée à la nutrition et à la santé des os

Près d'un quart des participants estimaient qu'il n'était pas possible de motiver de jeunes adultes parce qu'ils n'étaient tout simplement pas intéressés. Comme une des participantes l'a déclaré, « [t]out dépend d'eux. S'ils ne veulent pas faire ceci, même s'ils ont des connaissances, ils ne feront pas ce qu'il faut (femme, 23 ans, étudiante au College, P 46) ». Les participants qui jugeaient que les messages figurant sur les étiquettes pourraient être améliorés ont proposé que l'information soit explicative $(n=28)$ et pertinente $(n=30)$. «Je pense que si tu me disais quelque chose, je me demanderais comment ça influerait sur ma santé. Par exemple, si tu me disais que ça m'aiderait à perdre du poids ou à prendre de la force et que tu me donnais des exemples, je pense que ça attirerait plus mon attention et celle des gens. (femme, 17 ans, non étudiante, P 85)

La majorité des participants étaient d'avis que les jeunes adultes n'avaient pas assez de connaissances pour faire des choix éclairés et qu'il était important de leur dire pourquoi certains aliments ou certains comportements sont bénéfiques. «Je pense qu'on devrait en apprendre plus là-dessus au secondaire. Ils nous disent, genre, juste les mêmes choses, comme ce que j'ai dit tantôt, comme mange cette quantité de cela dans une journée. Mais ils ne te disent jamais vraiment quels sont les réels bénéfices des choses ni tous les différents types d'aliments. " (femme, 18 ans, étudiante à l'université, P 2). Les participants souhaiteraient que l'information offre des explications au lieu de présenter uniquement des listes d'aliments et de comportements.

Outre le fait qu'ils désiraient obtenir plus d'explications, les participants pensaient que les types de changements proposés devaient pouvoir s'intégrer aux vies occupées de jeunes adultes. Au lieu d'insister sur de grands changements à effectuer, un tiers des participants ont proposé de petites modifications simples et faciles à ajouter aux activités quotidiennes. "Je suis tout le temps étonné de voir comment de petites choses peuvent entraîner des changements. Quelque chose d'aussi simple qu'une campagne médiatique pourrait changer une petite habitude que tu garderais pour le reste de ta vie. " (homme, 28 ans, étudiant à l'université, P 30).
Si tous les participants étaient en faveur de campagnes de sensibilisation à l'ostéoporose et à l'information nutritionnelle liées à la santé des os, il y avait divergence d'opinions au sujet de la formulation des messages. Certains préconisaient l'emploi de messages suscitant la peur comme outil de persuasion : " Je pense qu'on doit sortir des sentiers battus ici. Genre, je sais qu'on ne devrait pas faire ça, mais le principe de la peur fonctionne assez bien. Si on montrait d'une certaine façon ce qu'est l'ostéoporose, à quel point ça peut être épeurant, dans quelle mesure ça peut influer sur ta vie. C'est tellement facile de la prévenir : il faut juste boire un verre de lait. » (femme, 22 ans, étudiante au College, $P$ 43). D'autres croient qu'utiliser la peur ne serait pas efficace à long terme : "Je sais que si tu montres toujours la maladie à quelqu'un ou que t'en parles avec une musique de fond triste, tu touches toujours la corde sensible de quelqu'un, mais ça sera oublié. " (homme, 22 ans, diplômé, P 87). Pour eux, il existe trop de maladies et les jeunes adultes sont insensibles aux messages jouant sur la peur. Un quart des participants a proposé l'utilisation de messages incitatifs ou positifs.

La multitude de renseignements disponibles semblant rendre le filtrage difficile, la plupart des participants estimaient que l'information serait plus accessible si elle était présentée dans des sources auxquelles ils avaient déjà accès. "Je crois qu'il faut nous joindre là où on se sent le plus à l'aise, ça pourrait être par l'université ou Facebook, des choses avec lesquelles vous savez qu'on sera en interaction. " (femme, 21 ans, étudiante à l'université, $P$ 18). La plupart des participants ont fait valoir que les médias sociaux constituent une source importante d'information, étant donné que presque tous les utilisent. Des réseaux sociaux populaires, comme Facebook, YouTube et Twitter ont été suggérés comme moyens efficaces de diffusion d'information. " Je dirais que ça devrait être des moyens auxquels ils sont grandement attachés. Je dirais des réseaux sociaux, des applications sur le téléphone. Ça devrait être des trucs liés à la technologie parce que si ce sont juste des affiches, les gens passent sans les voir parce qu'ils sont en train de texter. " (femme, 27 ans, étudiante au College, P 23). Le fait que la plupart des participants aient approuvé l'utilisation des médias sociaux est intéressant parce qu'ils les avaient identifiés auparavant comme une source problématique et non fiable. Il a été fréquemment 
mentionné que les approches axées sur la technologie et les médias sociaux en général constituaient le principal moyen d'accès à l'information. L'importance des témoignages de vedettes a été soulignée également par un petit nombre de participants $(n=5)$ : les célébrités sont suivies en ligne par un grand nombre de personnes et elles ont de l'influence sur les décisions des jeunes adultes. "Si tu essaies vraiment que ça devienne une cause importante, il y a alors les témoignages de vedettes, va chercher les bonnes personnes, les bons visages derrière elle. Va chercher les bonnes personnes pour la publier sur Twitter. Je parie que si t'avais Kim Kardashian pour commencer à appuyer la cause de l'ostéoporose, tu aurais probablement la moitié de l'Amérique du Nord au courant de ce qu'est l'ostéoporose. " (homme, 26 ans, étudiant au College, P 49).

\section{Analyse}

Les résultats de notre étude montrent que l'éducation à la prévention de l'ostéoporose en Ontario devrait s'inscrire dans une démarche de communication à l'intention des jeunes adultes qui s'articule autour de courts messages personnalisés, diffusés par l'entremise de réseaux traditionnels comme émergents et axés sur des changements à petite échelle pouvant être apportés dans les sphères d'intérêt de cette population. À l'instar des autres études, les jeunes participants adultes ont effectué sur Internet la majeure partie de leurs recherches ${ }^{33,34}$. Cependant, certains d'entre eux se sont dits encore influencés par leurs parents, les médecins, les éducateurs et les communautés de pairs. Même les plus âgés ayant quitté la résidence familiale ont déclaré se tenir informés grâce à leurs parents. Ces résultats recoupent ceux d'autres publications portant sur les habitudes de recherche d'information sur la santé et qui décrivent les médecins, les parents et les pairs comme des acteurs influents dans la vie des jeunes adultes ${ }^{33,35}$. La propension accrue observée chez les femmes à se fier à leur médecin pour obtenir une part importante de leur information reflète aussi certaines tendances en recherche voulant que les femmes soient davantage portées à consulter les professionnels de la santé que les hommes ${ }^{36}$. Les participants ont estimé que leurs parents et les professionnels de la santé sont des sources d'information fiables en raison des relations personnelles et professionnelles qu'ils entretiennent avec eux. Afin de tirer le meilleur parti de cette voie d'application des connaissances, les parents des adolescents et des jeunes adultes devraient eux aussi être renseignés au sujet de l'ostéoporose, notamment en ce qui a trait aux bienfaits à long terme de la prise de calcium et de vitamine $\mathrm{D}$. Comme les participants se sont dit réceptifs aux préoccupations soulevées par le personnel médical, inciter les médecins à parler du rôle clé de l'alimentation pour le maintien de la santé osseuse contribuerait aussi à une meilleure sensibilisation des jeunes adultes à la maladie. Par ailleurs, les études ont montré que les médecins de famille en Ontario accusent d'importantes lacunes de connaissances sur l'ostéoporose ${ }^{18,37}$, ce qui fait qu'améliorer la formation des médecins et les encourager à fournir des renseignements d'ordre préventif à leurs patients sans que ces derniers les sollicitent favoriserait l'assimilation des connaissances sur l'ostéoporose par les jeunes adultes.

Le rôle majeur des réseaux de pairs, en particulier celui des communautés en ligne, s'observe dans la confiance qu'accordent les jeunes adultes à Internet comme outil de recherche. Les groupes de pairs exercent une forte influence sur leur vie, et l'opinion et les pressions sociales des pairs façonnent leurs décisions ${ }^{38,39}$. Promouvoir la diffusion d'information sur la composante alimentaire de l'ostéoporose au sein des groupes de pairs dans les médias sociaux permettrait de sensibiliser un grand nombre de jeunes adultes. Les participants ont admis ne pas souvent chercher de façon active des renseignements relatifs à l'alimentation, mais ont précisé que les contenus informatifs intéressants captent facilement leur attention. Disséminer des renseignements par l'entremise de ces réseaux en ligne, surtout s'ils sont jumelés avec des contenus déjà recherchés par les jeunes adultes (p. ex. la mise en forme, la perte de poids, une saine alimentation, des faits intéressants), devrait accroître la visibilité et l'assimilation de ceux-ci.

Les médias sociaux sont devenus des outils essentiels de sensibilisation aux enjeux de santé. Toutefois, l'intérêt engrangé par les titres ou par les sujets demeure l'unique moyen pour ces sites d'attirer les utilisateurs qui ne se tiennent pas activement informés sur les questions de santé. Certains types de médias sociaux (Facebook, Twitter) accumulent une masse considérable d'information mise ligne par un nombre croissant de réseaux de pairs, mode de fonctionnement qui accélère la diffusion d'information auprès des utilisateurs n'étant pas à la recherche d'un contenu précis, mais tend en contrepartie à noyer les publications isolées dans ce flot d'informations ${ }^{40}$. Pour d'autres médias comme les blogues ou YouTube, les utilisateurs doivent chercher activement un contenu connexe, ce qui restreint la possibilité d'atteindre ceux qui ne cherchent pas d'information relative à la santé ou à l'alimentation ${ }^{40}$. Cependant, ces types de plateformes de diffusion entre pairs ont des effets favorables, comme en témoigne le récent succès du "défi du seau d'eau glacée» (Ice Bucket Challenge en anglais) au profit de la cause de la sclérose latérale amyotrophique (SLA) ${ }^{41,42, \dagger}$, et même si la portée dans le temps de ce travail de sensibilisation reste encore à évaluer. L'appui de célébrités, ce qui a suscité un intérêt et une émulation généralisés, explique en partie un tel succès, ce dont les participants de cette étude étaient consients ${ }^{43}$. Concevoir des messages d'ordre préventif diffusés en ligne requiert qu'une plus grande attention soit portée aux titres et aux images accrocheurs, et que ces connaissances soient présentées dans une forme adaptée au public cible.

Les raisons qui poussent les jeunes adultes à s'informer au sujet de l'alimentation et de la santé sont déterminantes dans la façon dont cette information leur est communiquée. Les notions de santé et d'alimentation ont été abordées séparément par les participants : les recherches sur les questions de santé étaient orientées vers le traitement des maladies, alors que celles portant sur l'alimentation visaient l'amélioration d'une santé déjà bonne. Si tous les participants ont associé alimentation et santé, leurs recherches relatives à la santé visaient à se renseigner sur des symptômes et étaient donc pour eux de plus grande importance parce qu'elles répondaient à un besoin de traitement. Leurs recherches portant sur l'alimentation, pour leur part, ont été associées à la prévention et étaient vues comme utiles au mieux-être, mais non essentielles. Les participants ont déclaré se préoccuper de

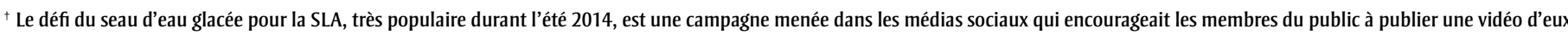

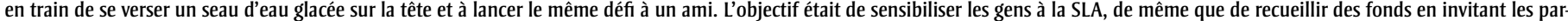
ticipants à faire un don. La campagne est rapidement devenue virale après avoir a été largement diffusée dans les médias sociaux et avoir reçu l'appui de célébrités.
} 
questions d'alimentation surtout en lien avec d'autres champs d'intérêt ou parce qu'il s'agissait d'un moyen d'atteindre des objectifs qui leur tenaient à cœur comme soigner son apparence, se mettre en forme, perdre du poids ou améliorer sa santé. Ces constats, déjà bien établis dans d'autres études portant sur les jeunes et la motivation à de saines habitudes alimentaires ${ }^{44-47}$, n'ont rien de surprenant : en effet, le plan établi par Santé Canada pour l'adoption de saines habitudes alimentaires, Bien manger avec le Guide alimentaire canadien, est conçu pour faire la promotion de l'alimentation en tant que moyen de lutte contre les maladies chroniques et l'obésitée ${ }^{\text {. }}$.

Les petites différences entre hommes et femmes observées dans le genre de renseignements recherchés témoignent de motivations distinctes pour les deux sexes en matière d'alimentation et de santé des os. Les différences dans la manière dont chacun des sexes de notre échantillon conçoit la santé des os, l'alimentation et la prévention de l'ostéoporose sont traitées ailleurs ${ }^{30}$. Il ressort des résultats de notre étude que les intervenants devraient créer une variété de messages adaptés tant aux champs d'intérêt des jeunes hommes qu'à ceux des jeunes femmes adultes. Les antécédents de maladie se sont par ailleurs révélés un facteur déterminant dans les habitudes de recherche d'information sur la santé et sur l'alimentation, et qui nécessite un examen plus approfondi.

Les résultats montrent en outre que la promotion de l'éducation en matière d'alimentation ne devrait pas se faire en référence à certaines maladies, mais s'intégrer aux divers sujets se rapportant aux habitudes alimentaires des jeunes adultes. Comme leurs choix ne reposent pas foncièrement sur des préoccupations liées à des maladies ou à la santé, l'information relative à l'ostéoporose devrait être incluse à celle qu'ils recherchent déjà. Ainsi, au lieu de mettre l'accent sur la sensibilisation à la maladie, l'information devrait être présentée sous l'angle de la perte de poids ou de la mise en forme en tant que renseignements susceptibles de les aider à devenir forts et en santé. Une approche de sensibilisation axée sur le rôle d'une bonne santé osseuse dans l'entraînement musculaire ou pour améliorer son apparence rejoindrait les préoccupations des jeunes adultes. De même, l'information sur la santé des os devrait figurer dans les sources de connaissances indiquées de sorte que les jeunes adultes sensibles à la teneur nutritive des aliments ou plus largement préoccupés par les systèmes alimentaires y aient accès.

L'abondante information axée sur les maladies mise à la disposition des jeunes adultes, en particulier sur Internet, peut désorienter les utilisateurs ${ }^{49}$. Des messages courts permettent de décomposer les renseignements complexes en des faits facilement assimilables. Inclure dans ces courts messages des conseils simples pour introduire des habitudes favorisant la santé osseuse dans le quotidien les rendrait d'autant plus attirants pour les jeunes adultes. Des exposés concis et simples portant sur le calcium, la vitamine D et la santé des os qui expliquent, par exemple, les bienfaits d'une consommation accrue de produits laitiers ou de légumes à feuilles foncées, sont plus à même de retenir l'attention des jeunes adultes qui s'estiment trop occupés pour opérer d'importants changements dans leur mode de vie.

Délaisser une approche de communication orientée sur les maladies implique aussi de limiter le recours aux campagnes alarmistes au profit de messages qui incitent à l'action. Qui plus est, fournir aux jeunes adultes les raisons de consommer du calcium et de la vitamine D pour s'assurer une bonne santé osseuse rend ces derniers plus enclins à prêter attention aux messages et à les retenir. Ne pouvant s'appuyer sur un raisonnement causal clair, les participants de cette étude ont pu difficilement expliquer l'intérêt qu'ils auraient à modifier leur alimentation ainsi que conceptualiser les conséquences de leurs habitudes sur leur risque de développer certaines maladies. Une approche de communication qui leur apporterait des raisons concrètes et qui rattacherait l'information à des aspects de leur santé physique qui les préoccupent serait probablement plus efficace puisqu'elle a recours à des messages positifs plutôt que négatifs pour encourager au changement.

Tout compte fait, l'alimentation constitue un véhicule important pour transmettre aux jeunes adultes des renseignements sur la prévention de l'ostéoporose. Les changements dans l'alimentation s'intègrent facilement dans la vie de tous les jours, et présenter les messages d'éducation à l'ostéoporose dans ce cadre permettrait de recouper les sphères d'intérêt de cette population. Établir des liens entre les habitudes alimentaires et les problèmes de santé pourrait encourager la prévention de l'ostéoporose et possiblement réduire les taux de la maladie dans l'avenir.

\section{Points forts et limites}

Notre étude examine de manière rigoureuse et qualitative l'application des connaissances sur l'ostéoporose au sein d'un échantillon représentatif de la population de jeunes adultes de l'Ontario. Un échantillon de petite taille a été utilisé en raison du caractère qualitatif de l'étude, ce qui réduit la possibilité de généraliser les résultats pour une plus grande population, mais renseigne sur le contexte du sud de l'Ontario. Les deux tiers des participants ont été recrutés dans des établissements postsecondaires, ce qui pourrait introduire un biais en faveur des participants dont le niveau d'instruction est plus élevé. Ces chiffres reflètent néanmoins les taux d'inscription aux études postsecondaires en Ontario. Les données recueillies au cours des entrevues ont été fournies par les participants eux-mêmes, ce qui ouvre la porte à des oublis ou à des difficultés de remémoration. En raison du petit nombre de participants dans chaque catégorie d’âge, il n'a pas été possible d'examiner de différences relatives à cette variable au sein de l'échantillon à l'étude. De plus, le codage et l'analyse ont été réalisés par l'auteur seul et pourraient être une source de biais.

\section{Conclusion}

Pour diminuer la prévalence future de l'ostéoporose, il faut réduire dès aujourd'hui le risque de développer cette maladie chez les jeunes adultes, qui ont encore la possibilité de freiner leur perte osseuse. Mettre sur pied des programmes efficaces d'éducation à la prévention de l'ostéoporose destinés aux jeunes adultes demande que l'information leur soit communiquée de façon à ce qu'ils puissent la reconnaître, $\mathrm{y}$ accéder et en tirer profit dans leur vie. L'examen de leurs habitudes a mis en évidence que même s'ils disposent de vastes réseaux d'information, les jeunes adultes recherchent rarement activement des renseignements sur le rôle de l'alimentation dans la prévention de l'ostéoporose. Les sensibiliser à l'ostéoporose afin de les encourager à adopter une attitude préventive pourrait se faire plus efficacement si ces messages éducatifs se rattachaient aux autres formes d'information consultées. Leur transmettre des connaissances exige que l'information soit présentée de manière 
à établir un lien clair entre l'ostéoporose et l'apparence corporelle, que les médias en ligne soient mis à contribution pour assurer leur diffusion et que l'approche soit aussi centrée sur des réseaux plus traditionnels (parents, médecins), parfois sous-estimés à l'ère numérique mais demeurant des sources d'information influentes et fiables pour les jeunes adultes ayant pris part à l'étude. L'information relative à la prévention de l'ostéoporose devrait prendre la forme de messages encourageant des changements de style de vie pratiques, à petite échelle et spécifiquement ciblés pour les jeunes adultes, messages accompagnés d'explications. Cette étude propose déjà certaines modifications aux programmes de prévention, sachant qu'il faudra par la suite élaborer une stratégie de communication spécifiquement destinée aux jeunes adultes et dont l'efficacité pourra être évaluée.

\section{Remerciements}

Cette étude a été financée par le Régime de bourses d'études supérieures de l'Ontario. Ma superviseuse, $\mathrm{D}^{\mathrm{re}}$ Tina Moffat, ainsi que les membres de mon comité, $\mathrm{D}^{\text {re }}$ Megan Brickley et $\mathrm{D}^{\text {re }}$ Stephanie Atkinsion, ont fourni leur aide et leurs conseils lors de la conception et de la mise en œuvre de ce projet.

\section{Conflits d'intérêts}

L’auteure déclare n'avoir aucun conflit d'intérêts.

\section{Références}

1. Kanis J on behalf of the World Health Organization Scientific Group. Assessment of osteoporosis at the primary health care level. Sheffield (UK): World Health Organization Collaborating Centre for Metabolic Bone Diseases, University of Sheffield; 2007. [Technical report]. $339 \mathrm{p}$.

2. Tarride JE, Hopkins RB, Leslie WD, et al. The burden of illness of osteoporosis in Canada. Osteoporos Int. 2012; 23(11):2591-2600.

3. Ostéoporose Canada. Données et statistiques [Internet]. Toronto (Ont.) : Ostéoporose Canada; 2017 [consulté en février 2016]. En ligne à : http:// www.osteoporosecanada.ca/losteoporose -et-vous/donnees-et-statistiques -sur-losteoporose/
4. Organisation mondiale de la Santé (OMS). Prevention and management of osteoporosis. Genève $(\mathrm{CH})$ : OMS; 2003. [Série de rapports technique $\mathrm{n}^{\circ}$ 921].

5. Cooper C, Cole ZA, Holroyd CR, et al. Secular trends in the incidence of hip and other osteoporotic fractures. Osteoporos Int. 2011;22(5):1277-1288.

6. Kasper MJ, Peterson MG, Allegrante JP. The need for comprehensive educational osteoporosis prevention programs for young women: results from a second osteoporosis prevention survey. Arthritis Rheum. 2001;45(1):28-34.

7. Tussing L, Chapman-Novakofski K. Osteoporosis prevention education: behaviour theories and calcium intake. J Am Diet Assoc. 2005;105(1):92-97.

8. Organisation mondiale de la Santé (OMS). Régime alimentaire, nutrition et prévention des maladies chroniques. Genève (CH) : OMS; 2003. 160 p. [Série de rapports techniques de l'OMS, $\left.n^{\circ} 916\right]$

9. Instituts de recherche en santé du Canada (IRSC). Stratégie liée à l'application des connaissances 2004-2009 [Internet]. Ottawa (Ont.) : IRSC; 2008 [consulté en février 2016]. En ligne à : http://www.cihr-irsc.gc.ca/f/26574 .html

10. Graham I, Logan J, Harrison M, et al. Lost in knowledge translation: time for a map? J Contin Educ Health Prof. 2006;26(1):13-24.

11. Straus SE, Tetroe J, Graham ID, editors. Knowledge translation in health care. Chichester (UK): John Wiley and Sons; 2013. 424 p.

12. Ostéoporose Canada. Briser les obstacles sans briser les os : Bulletin national 2008 sur les soins en santé en ostéoporose. Toronto (Ont.) : Ostéoporose Canada; 2008. 33 p.

13. Government of Manitoba. Manitoba falls prevention strategy and framework [Internet]. Winnipeg (MB) : gouvernement du Manitoba; 2006 [consulté en février 2016]. En ligne à : http:// www.gov.mb.ca/healthyliving/hlp /injury/falls.html
14. Nova Scotia Department of Health and Wellness. Halifax Osteoporosis Multidisciplinary Education (HOME) [Internet]. Halifax (NS) : Nova Scotia Department of Health and Wellness; 2016 [consulté en février 2016]. En ligne à : http://www.cdha.nshealth .ca/osteoporosis/halifax-osteoporosis -multidisciplinary-education-home

15. British Columbia Ministry of Health, British Columbia Medical Association. Osteoporosis: diagnosis, treatment and fracture prevention. Vancouver (BC) : Guidelines and Protocols and Advisory Committee; 2012. 17 p.

16. Ministère de la Santé et des Soins de longue durée de l'Ontario, Société de l'ostéoporose du Canada. Plan d'action contre l'ostéoporose : une stratégie de lutte contre l'ostéoporose en Ontario. Imprimeur de la Reine pour l'Ontario; 2003. 88 p. [ $\mathrm{n}^{\circ}$ de catalogue : 7610 $-3238905]$

17. Jaglal SB, Hawker G, Cameron C, et al. The Ontario osteoporosis strategy: implementation of a population-based osteoporosis action plan in Canada. Osteoporos Int. 2012;21(6):903-908.

18. Jaglal SB, Hawker G, Bansod V, et al. A demonstration project of a multicomponent educational intervention to improve integrated post-fracture osteoporosis care in five rural communities in Ontario, Canada. Osteoporos Int. 2009;20(2):265-274.

19. Baxter-Jones ADG, Faulkner RA, Forwood MR, Mirwald RL, Bailey DA. Bone mineral accrual from 8 to 30 years of age: an estimation of peak bone mass. J Bone Miner Res. 2011; 26(8):1729-1739.

20. Chan MF, Kwong WS, Zang Y, Wan P. Evaluation of an osteoporosis prevention education programme for young adults. J Adv Nurs. 2007;57(3): 270-285.

21. Neumark-Sztainer D, Story M, Perry C, Casey MA. Factors influencing food choices of adolescents: findings from focus-group discussions with adolescents. J Am Diet Assoc. 1999;99(8): 929-937. 
22. Burgener M, Arnold M, Katz JN, et al. Older adults' knowledge and beliefs about osteoporosis: results of semistructured interviews used for the development of education materials. J Rheumatol. 2005;32(4):673-677.

23. Doheny MO, Sedlak CA, Estok PJ, Zeller R. Osteoporosis knowledge, health beliefs, and DXA T-scores in men and women 50 years of age and older. Orthop Nurs. 2007:26(4):243-250.

24. Santé Canada. Les adultes canadiens comblent-ils leur besoins en nutriments uniquement grâce à l'alimentation? Ottawa (Ont.) : Santé Canada; 2012. 9 p. [n ${ }^{\circ}$ de catalogue : H164-112/ 3-2012F-PDF]

25. Chang S-F. A cross-sectional survey of calcium intake in relation to knowledge of osteoporosis and beliefs in young adult women. Int J Nurs Pract. 2006;12(1):21-27.

26. Ford MA, Bass MA, Keathley R. Osteoporosis knowledge and attitudes: a cross-sectional study among collegeage students. J Am Coll Health. 2007; 56(1):43-47.

27. Ziccardi SL, Sedlak CA, Doheny MO. Knowledge and health beliefs of osteoporosis in college nursing students. Orthop Nurs. 2004;23(2):128-133.

28. Butow P, Palmer S, Pai A, Goodenough $B$, Luckett T, King M. Review of adherence-related issues in adolescents and young adults with cancer. J Clin Oncol. 2010;28(32):4800-4849.

29. Geiger A, Castellino SM. Delineating the age ranges used to define adolescents and young adults. J Clin Oncol. 2011;29(16):e492-e493.

30. Holland A. Thwarting the silent thief: informing nutrition-based osteoporosis prevention education for young adults [dissertation]. Hamilton (Ont.) : McMaster University; 2016. 229 p.

31. Braun V, Clarke V. Using thematic analysis in psychology. Qual Res Psychol. 2006;3(2):77-101.

32. Bernard H. Research methods in anthropology: qualitative and quantitative approaches. 5e éd. Maryland (UK) : AltaMira Press; 2011. 680 p.
33. Gray NJ, Klein JD, Noyce PR, Sesselberg TS, Cantrill JA. Health information-seeking behavior in adolescence: the place of the internet. Soc Sci Med. 2005;60(7):1467-1478.

34. Hesse BW, Nelson DE, Kreps GL, et al. Trust and sources of health information: the impact of the internet and its implications for health care providers: findings from the first Health Information National Trends Survey. Arch Intern Med. 2005;165(22): 2618-2624.

35. Canadian Council of Food and Nutrition (CCFN). Tracking nutrition trends: a 20-year history. Ottawa (Ont.) : CCFN; 2009. En ligne à : http://www.cfdr.ca/Downloads /CCFN-docs/20-Years-of-TNT-(Sep12) ---Final.aspx

36. Thompson AE, Anisimowicz Y, Miedema B, Hogg W, Wodchis WP, Aubrey-Bassler K. The influence of gender and other patient characteristics on health care-seeking behaviour: a QUALICOPC study. BMC Fam Pract. 2016;17:38. doi: 10.1186/s12875-016 $-0440-0$.

37. Jaglal SB, McIsaac WJ, Hawker G, et al. Information needs in the management of osteoporosis in family practice: an illustration of the failure of the current guideline implementation process. Osteoporos Int. 2003;14(8): 672-676.

38. Kelder S, Perry CL, Klepp KI, Lytle LL. Longitudinal tracking of adolescent smoking, physical activity, and food choice behaviors. Am J Public Health. 1994;84(7):1121-1126.

39. Story M, Neumark-Sztainer D, French S. Individual and environmental influences on adolescent eating behaviors. J Am Diet Assoc. 2002;102(3 Suppl): S40-S51.

40. Korda H, Itani Z. Harnessing social media for health promotion and behavior change. Health Promot Pract. 2013;14(1):15-23.

41. Carrì MT. Philanthropy: ice bucket challenge should jolt funding [letter]. Nature. 2014;515(7527):343.
42. Koohy H, Koohy B. A lesson from the ice bucket challenge: using social networks to publicize science. Front Genet. 2014;5:430. doi: 10.3389/fgene .2014 .00430 .

43. Ni MY, Chan BHY, Leung GM, Lau EHY, Pang H. Transmissibility of the Ice Bucket Challenge among globally influential celebrities: retrospective cohort study. BMJ. 2014;349:g7185. doi: 10.1136/bmj.g7185.

44. Betts NM, Amos RJ, Keim KS, Peters P, Stewart B. Ways young adults view foods. J Nutr Educ. 1997;29(2):73-79.

45. Counihan CM. Food rules in the US: individualism, control, and hierarchy. Anthropol Q. 1992;65(2):55-66.

46. Ousley L, Cordero ED, White S. Fat talk among college students: how undergraduates communicate regarding food and body weight, shape and appearance. Eat Disord. 2008;16(1): 73-84.

47. Rozin P, Bauer R, Catanese D. Food and life, pleasure and worry, among American college students: gender differences and regional similarities. J Pers Soc Psychol. 2003;85(1):132-141.

48. Bush MA, Martineau C, Pronk JA, Brulé D. Eating well with Canada's food guide: "a tool for the times". Can J Diet Pract Res. 2007;68(2):92-96.

49. Murero M, Rice R, editors. The internet and health care: theory, research, and practice. Mahwah (NJ): Lawrence Erlbaum Associates; 2006. 403 p. 